\title{
Relación entre condición física global, coordinación motriz y calidad de vida percibida en adolescentes españoles
}

\author{
Eliseo García Cantó; Andrés Rosa Guillamón; Laura Nieto López
}

Cómo citar este artículo:

García Cantó, E., Rosa Guillamón, A., \& Nieto López, L. (2021). Relación entre condición física global, coordinación motriz y calidad de vida percibida en adolescentes españoles. Acta Colombiana de Psicología, 24(1), 96-106. https://www.doi. org/10.14718/ACP.2021.24.1.9

Recibido, noviembre 12/2019; Concepto de evaluación, julio 27/2020; Aceptado, agosto 18/2020

\author{
Eliseo García Cantó ${ }^{1}$ \\ ORCID: https://orcid.org/0000-0002-6845-6835 \\ Universidad de Murcia, Murcia, España. \\ Andrés Rosa Guillamón \\ ORCID: https://orcid.org/0000-0001-5679-0986 \\ Universidad de Murcia, Murcia, España. \\ Laura Nieto López \\ ORCID: https://orcid.org/0000-0003-4624-4054 \\ Universidad de Murcia, Murcia, España.
}

\begin{abstract}
Resumen
La sociedad actual plantea la necesidad de incorporar a la educación aquellas capacidades relacionadas con el cuerpo y su actividad motriz dentro de los márgenes de la salud, pues estas resultan determinantes para el desarrollo integral de la persona. Teniendo esto en cuenta, el objetivo del presente estudio fue analizar la relación entre la condición física global (CF), la coordinación motriz (CM) y la calidad de vida percibida (CVP) en adolescentes españoles. Para esto, se diseñó un estudio descriptivo transversal con 1037 adolescentes de 14 a 16 años de edad, y se utilizó el Cuestionario de salud SF-36 para evaluar la CVP, y la Batería de condición fisica relacionada con la salud para evaluar la CF global y la CM. Los resultados muestran que los sujetos con mayor CF tuvieron valores promedio más elevados en las variables de función física $(p<.001)$, rol físico $(p<.001)$, vitalidad $(p<.05)$, función social $(p<.001)$, rol emocional $(p<.01)$, salud mental $(p<.001)$ y calidad de vida total $(p<.001)$; mientras que aquellos con menor CF presentaron puntuaciones superiores en dolor corporal $(p<.001)$. Asimismo, los sujetos que mostraron mayor $\mathrm{CM}$ obtuvieron mejores registros en salud general, vitalidad, función social y calidad de vida total $(p<.001$ en todos los casos), y registros inferiores en función física y rol físico $(p<.001$ en ambos casos). Los resultados de este estudio sugieren que tener una mayor CF, así como una mayor CM, pueden ser variables predictoras de una mejor CVP en adolescentes españoles.

Palabras clave: condición física global, coordinación motriz, calidad de vida percibida.
\end{abstract}

\section{Relationship between overall physical condition, motor coordination and perceived quality of life in Spanish adolescents}

\begin{abstract}
Today's society poses the need to incorporate into education those capacities related to the body and its motor activity within the margins of health, since these are determining factors for the integral development of the person. Bearing this in mind, the aim of this study was to analyze the relationship between overall physical condition (PC), motor coordination (MC) and perceived quality of life (PQL) in Spanish adolescents. For this purpose, a cross-sectional descriptive study was designed
\end{abstract}

C/Doctor Ricardo Cano 14, 2 a. Teléfono: +34 627544824. Correo electrónico: eliseo.garcia@um.es 
involving 1037 adolescents aged between 14 to 16 years. The SF-36 Health Questionnaire was used to assess the PQL, and the Health-related Physical Condition Battery, to evaluate global PC and MC. The results show that participants with higher PC had higher average values in the variables physical function $(\mathrm{p}<.001)$, physical role $(\mathrm{p}<.001)$, vitality $(\mathrm{p}<.05)$, social function $(p<.001)$, emotional role $(p<.01)$, mental health $(p<.001)$, and total quality of life $(p<.001)$; while those with lower PC had higher scores in body pain $(\mathrm{p}<.001)$. Likewise, participants who showed higher MC obtained better records in general health, vitality, social function, and total quality of life $(\mathrm{p}<.001$ in all cases), and lower records in physical function and physical role ( $\mathrm{p}<.001$ in both cases). The results of this study suggest that having a higher PC, as well as a higher MC, maybe predictive variables of a better PQL in Spanish adolescents.

Keywords: global physical condition, motor coordination, perceived quality of life.

\section{Introducción}

El estudio de aspectos relacionados con la competencia motriz y la calidad de vida percibida (CVP) de un individuo ha sido objeto importante de investigación en los últimos años, debido a la necesidad de la sociedad actual de incorporar a la educación aquellas capacidades relacionadas con el cuerpo y su actividad motriz, teniendo en cuenta que ambas son determinantes para el desarrollo integral de la persona y, por ende, para la mejora de su calidad de vida (Fort-Vanmeerhaeghe et al., 2017; Gil et al., 2020; Rosa et al., 2018). En este sentido, resulta interesante remarcar la idea de que, para lograr una mayor calidad de vida, pueden ser importantes los programas de prevención que tienen como base la actividad física y el desarrollo de la condición física (CF) (Mastrantonio \& Coduras, 2020; Moral-García et al., 2019).

Así pues, según diversos autores (Gálvez et al., 2015a), la capacidad para realizar actividad física es uno de los factores con mayor influencia sobre el nivel de CF de un individuo, y a partir de su valoración es posible extraer datos interesantes respecto al estado de las funciones relacionadas con la práctica de actividad motriz (Nieto et al., 2020; Ruiz et al., 2009), pues los principales indicadores de una CF orientada a la salud suelen ser la composición corporal, la capacidad motora, la capacidad aeróbica y la fuerza músculo-esquelética (Rosa-Guillamón, 2017; RosaGuillamón et al., 2020).

Siguiendo en esta línea, la CF global de un individuo se convierte uno de los pilares en los que se basa otro de los constructos a analizar en nuestro estudio, la coordinación motriz (CM), pues esta se ha configurado como uno de los determinantes más importantes para el bienestar del adolescente desde una perspectiva física y mental (Ramón \& Ruiz-Pérez, 2015). Teniendo esto en consideración, se ha encontrado que el desarrollo de la CM es un aspecto fundamental para lograr hábitos favorables para la adquisición de un estilo de vida saludable basado en la realización de actividad física (FortVanmeerhaeghe et al., 2017; Rosa et al., 2020).
De hecho, se ha hallado que niveles altos de cm podrían influir positivamente y de manera indirecta en la CVP, dada la importancia de las conductas que adopta el adolescente en su interacción con el entorno, en sus hábitos psicosociales, y en el desarrollo de otras dimensiones de su personalidad —como la autopercepción de competencia, por ejemplo(Ramón \& Ruiz-Pérez, 2015).

En particular, el desarrollo psicosocial es considerado una dimensión fundamental en la etapa adolescente, de ahí que cada vez existan más investigaciones que busquen analizar las relaciones entre la CM y aspectos relacionados con la percepción de la salud física y psicológica del sujeto, su nivel de independencia, sus relaciones sociales (Rigoli et al., 2012; Robinson et al., 2015), e incluso su cognición y su rendimiento académico (Guillamón et al., 2020; Ortiz \& Ramírez, 2020; Ruiz-Pérez et al., 2016).

En este sentido, se ha encontrado que el nivel de cvp está condicionado por los procesos cognitivos vinculados a la evaluación que la persona realiza tanto de sus condiciones objetivas de vida como del grado de satisfacción con cada una de ellas (Baceviciene et al., 2019); e, incluso, que la calidad de vida es subjetiva, que el valor asignado a cada dimensión es diferente en cada persona, y que este valor puede cambiar a través del tiempo (Urzúa \& Caqueo-Urizar, 2012). Por consiguiente, algunos autores han enfatizado en la necesidad de profundizar en los indicadores, desórdenes psicosociales y experiencias vinculadas a la CVP en la etapa adolescente (Salinas-Miranda et al., 2015; Salum et al., 2014), sobre todo por ser esta una interesante medida del estado de salud desde la perspectiva del propio sujeto (Herranz et al., 2013).

En cambio, otras investigaciones apuntan a diversos indicadores del estado de salud relacionado con la CF y a la CF global (Becerra et al., 2013; Rosa-Guillamón et al., 2016; Rosa-Guillamón et al., 2018), donde se sugiere que la capacidad aeróbica, la fuerza muscular o el índice de masa corporal (IMC) pueden suponer elementos diferenciadores de la CVP y la satisfacción con la vida desde edades tempranas (Andersen et al., 2017; Evaristo et al., 2019; 
Gálvez et al., 2015b; Guedes et al., 2017; Marques et al., 2017; Mota et al., 2012).

En esta línea de ideas, en el estudio de Baceviciene et al. (2019) se concluyó que un mejor nivel de CF global se asocia a una mayor CVP en adolescentes con estilos de vida no saludables, y en el trabajo de Wu et al. (2017) se indica que la práctica habitual de actividad física podría asociarse a una mejor CVP. De hecho, este último aspecto había sido abordado en un estudio previo en el que se reportó que la relación entre la práctica física habitual y el IMC con la CVP se encuentra mediada por el sexo, la edad y la cF global de los participantes (García-Rubio et al., 2015).

No obstante, y a pesar de estos hallazgos, la literatura científica no se muestra concluyente en el estudio de la relación entre la CF y la CVP en adolescentes (Baceviciene et al., 2019). Además, son escasos los estudios que analizan la relación entre la CVP y la CM (Caçola \& Killian, 2018; Rigoli, \& Piek, 2016); siendo esta última una variable fundamental para que los adolescentes dispongan de un mayor repertorio de opciones motrices en las que se sientan competentes; lo que a la vez genera más adherencia a la actividad física y, en consecuencia, una mejor CF (Karras et al., 2019; Zwicker et al., 2018).

Con base en lo anterior, en el presente estudio se plantearon dos hipótesis: (a) que a mayor nivel de condición física, mayor CVP; y (b) que a mayor nivel de coordinación motriz, mejor CVP. Por tanto, el objetivo principal de este trabajo fue analizar la relación entre la CF, la CM y la CVP en adolescentes españoles, así como observar la capacidad predictiva tanto de la CF como de la CM sobre la CVP.

\section{Método}

\section{Tipo de estudio, diseño y participantes}

Se realizó un estudio descriptivo de corte transversal ex post facto (Montero \& León, 2002) con 1037 adolescentes españoles con una media de edad de $15.52( \pm 1.11)$ años. Para abarcar la representatividad de la población, el estudio se desarrolló en diversos centros públicos y privados de Educación Secundaria Obligatoria y Bachillerato de la Región de Murcia, en España.

Todos los participantes entregaron el consentimiento informado de sus padres, madres o tutores legales; y los criterios de exclusión fueron presentar enfermedades que pudieran suponer un riesgo ante el esfuerzo físico intenso, así como informar presencia de enfermedades mentales.

El estudio se realizó según las normas deontológicas reconocidas por la Declaración de Helsinki (revisión de 2013), las recomendaciones de Buena Práctica Clínica de la CEE (documento 111/3976/88 de julio de 1990), y la normativa legal española para la investigación clínica en humanos (Real Decreto 561 de 1993 sobre ensayos clínicos). Además, el estudio se elaboró a partir del código ético de la Universidad de Murcia, y hace parte de una tesis doctoral desarrollada en el Departamento de Expresión Plástica, Musical y Dinámica de la Universidad de Murcia, España, aprobada por la comisión de doctorado de la Facultad de Educación de dicha universidad el 7 de septiembre de 2016.

\section{Variables e instrumentos}

\section{Calidad de vida percibida}

Para la evaluación de la CVP se utilizó el Cuestionario de salud SF-36, de Ware y Sherbourne (1992), un instrumento con valores adecuados de consistencia interna ( $\alpha$ de Cronbach $\geq .7$ ) a nivel internacional (Vilagut et al., 2005), diseñado para ser autoadministrado por personas con una edades superiores a los 14 años, y compuesto por 36 ítems que valoran ocho dimensiones de la salud -positivas y negativas - relacionadas con la CVP: función física, rol físico, dolor corporal, función social, rol emocional, salud mental, vitalidad, y salud general. Las opciones de respuesta se presentan en escalas tipo Likert — para evaluar intensidad o frecuencia-, y el número de opciones de respuesta varía entre 2,3 y 6 , según el ítem.

\section{Condición física y coordinación motriz}

Para evaluar la CF y la CM se administró la Batería de condición física relacionada con la salud (COFISA), de Lázaro-Martínez (2011), cuya fiabilidad, validez y protocolo de administración de las pruebas en adolescentes ha sido demostrada anteriormente (Lázaro-Martínez, 2011). Los test fueron administrados dos veces - excepto el test de Ruffier-, donde se registró un mejor rendimiento para la segunda medida en cada una de las pruebas. El test de Ruffier solo se realizó una vez debido a su elevada intensidad física y por la escasez de tiempo para administrar la batería de test en los centros educativos que hicieron parte del estudio.

En general, la variable CF global se calculó, con valores entre 0 y 10, como la suma de la media escalada de las puntuaciones de los participantes en los test de fuerza de prensión, abdominales, Ruffier y distancia dedos-plantas. Se categorizó a los participantes en dos grupos según su nivel de CF, ajustado por edad y sexo: menor CF (percentil $\leq 50$ ) y mayor $\mathrm{CF}$ (percentil $>50$ ); mientras que la variable CM se creó, con valores entre 0 y 10, como la suma de la media escalada de las puntuaciones de los participantes en los test de carrera de obstáculos, manejo de balón con 
las manos, manejo de balón con los pies y lanzamientorecepción. Asimismo, los participantes fueron clasificados en dos grupos según su nivel de CM, ajustado por edad y sexo: menor $\mathrm{CM}($ percentil $\leq 50)$ y mayor $\mathrm{CM}$ (percentil $>50$ ). Los test aplicados se describen a continuación.

Test de fuerza máxima de prensión $(\mathrm{kg})$.

Este test mide la fuerza máxima e isométrica de la musculatura flexora de la mano. Acá, los participantes tuvieron que permanecer de pie, con el brazo extendido, sin ninguna flexión, extensión o rotación de la mano. Se empleó un dinamómetro digital (ткK 5041 Grip D, Takei, Tokio, Japón), y una regla-tabla para adaptar la amplitud del agarre (España-Romero et al., 2010).

Test de abdominales (núm.).

Este test mide la fuerza-resistencia de la musculatura flexora del tronco. Acá, los participantes se colocaron en posición supina sobre una colchoneta dura de $1.5 \mathrm{~m} \times 1 \mathrm{~m} \times 0.8 \mathrm{~cm}$ con las rodillas en flexión de $90^{\circ}$. Se anotó el número (núm.) de encogimientos abdominales realizados durante 30 segundos.

Test de Ruffier (índice de Ruffier).

Este test mide la resistencia cardiorrespiratoria. Acá, los participantes tuvieron que ejecutar 30 flexo-extensiones completas de rodilla en un tiempo de 30-45 segundos, siguiendo el ritmo marcado por una señal sonora. El índice de Ruffier se calculó utilizando la fórmula: P+P'+ P' $-200 / 10$, donde $\mathrm{P}=$ pulsaciones en reposo (basal); $\mathrm{P}^{\prime}=$ pulsaciones al finalizar la prueba (adaptación); $\mathrm{P}$ " = pulsaciones al minuto de finalizar la prueba (recuperación). Se utilizó un pulsómetro con conectividad Bluetooth (Polar Electro Oy, H7, Kempele, Finlandia) para la medición de las pulsaciones.

Test de distancia dedos-plantas ( $\mathrm{cm}$ ).

Este test mide la flexibilidad de la musculatura lumbar e isquiosural. Acá, los participantes se colocaron con la cadera flexionada en ángulo recto con respecto a las extremidades, que se encontraban extendidas y juntas. Se empleó un cajón de $35 \mathrm{~cm} \times 45 \mathrm{~cm} \times 32 \mathrm{~cm}$, junto con una placa superior de $55 \mathrm{~cm} \times 45 \mathrm{~cm}$ de ancho que sobresale $15 \mathrm{~cm}$ del largo del cajón, y una regla móvil de $50 \mathrm{~cm}$ adosada a la placa superior. Se registraron los centímetros $(\mathrm{cm})$ que sobrepasaron (valores positivos) y los que no sobrepasaron (valores negativos) las puntas de los pies con las dos manos paralelas.

Test de coordinación motriz, circuito de agilidad (s).

Este test mide la velocidad-agilidad. Acá, los participantes tuvieron que completar un circuito de obstáculos y se evaluó el tiempo en segundos ( $s$ ) que tardaban en terminar el circuito. Se consideró como nulo el derribo de una pica, valla o modificar el recorrido establecido. Para la medición del tiempo se utilizó un cronómetro profesional (HS-80TW-1EF, Casio, Tokio, Japón).

Test de manejo de balón con las manos (s) y test de manejo de balón con los pies (s).

Estos test valoran la coordinación óculo-manual y la coordinación óculo-pedal, respectivamente. Las pruebas se desarrollan sobre un espacio de $20 \mathrm{~m} \times 4 \mathrm{~m}$, con seis picas insertadas sobre seis conos, colocados en línea, con una separación entre ellos de $2 \mathrm{~m}$. En el test de manejo de balón con las manos, los participantes tuvieron que realizar el recorrido en zigzag botando una pelota de voleibol con una o ambas manos. En el test de manejo de balón con los pies, los participantes tuvieron que realizar el recorrido en zigzag conduciendo una pelota de voleibol con uno o ambos pies. La pelota de voleibol tenía una circunferencia de $65 \mathrm{~cm}$, un peso de $260 \mathrm{~g}$ y una presión de entre 0.300 y $0.325 \mathrm{~kg} / \mathrm{cm}^{2}$. Para la medición del tiempo $(s)$, se utilizó un cronómetro profesional (HS-80TW-1EF, Casio, Tokio, Japón).

Test de lanzamiento-recepción (núm.).

Este test mide la coordinación óculo-manual, la precisión y la recepción. El espacio de práctica se establece sobre dos planos: uno vertical, con unas dimensiones de $3 \mathrm{~m} \times 3 \mathrm{~m}$, y otro horizontal, con unas dimensiones de $3 \mathrm{~m} \times 3 \mathrm{~m}$ de largo. Se dibujó en la pared un círculo de $40 \mathrm{~cm}$ de radio, con un borde de $5 \mathrm{~cm}$, a una altura de $1.60 \mathrm{~m}$ del suelo. En esta prueba, los participantes tuvieron que realizar el mayor número de lanzamientos-recepciones (secuencias) al círculo con una pelota de voleibol durante 30 segundos. Se anotó el número (núm.) de secuencias correctas.

\section{Variables de confusión controladas}

Se controlaron las variables de peso $(\mathrm{kg})$ y talla $(\mathrm{cm})$. El peso se midió utilizando una báscula electrónica (modelo 220, SECA, Hamburgo, Alemania) con un nivel de error de $\pm 0.1 \mathrm{~kg} ; \mathrm{y}$ la talla se midió en centímetros, empleando un tallímetro telescópico incorporado en la báscula. A partir de esto, se calculó el IMC, entendido como el peso en kilogramos dividido por la talla en centímetros al cuadrado.

\section{Análisis estadístico}

Las variables continuas se presentan como media, desviación estándar y error estándar, mientras que las variables categóricas se presentan como porcentajes y recuento numérico. Tras el análisis preliminar, se realizó un análisis de regresión lineal para estudiar la asociación entre la CM, la CF y la CVP, en el cual se hicieron los ajustes (covariables) por sexo $(0=$ mujeres, $1=$ varones $)$, edad (años) e IMC $\left(\mathrm{kg} / \mathrm{m}^{2}\right)$. Específicamente, para estudiar las diferencias en las dimensiones de la CVP, así como en su valor global, se realizaron análisis de la covarianza (ANCOVA) ajustados por sexo y edad; además, se realizaron 
los análisis para varones y mujeres conjuntamente para lograr una mayor potencia estadística. La significancia estadística fue establecida en un valor $p<.05$. Los cálculos fueron realizados con el programa SPSS (v.23.0 de SPSS Inc., Chicago, Illinois, EE. UU.).

\section{Resultados}

Como se muestra en la Tabla 1, los varones mostraron valores más elevados en rol emocional $(p=.010)$ y salud mental $(p<.001)$ que las mujeres, quienes presentaron valores superiores frente a los varones en salud general $(p=.011)$. Adicional a esto, en el rendimiento físico en los test, los varones presentaron valores superiores que las mujeres en todos los test de CF y CM $(p<.001)$, así como un mayor peso y talla $(p<.001)$; aunque las mujeres tuvieron un mejor rendimiento en el test de distancia dedos-plantas $(p<.001)$, así como una prevalencia superior, de mayor CM que los varones (54.8 vs. 45.2 ).

Por otra parte, en la Tabla 2 se presenta el análisis de regresión lineal múltiple ajustado por sexo, edad e IMC, en el cual se pone de manifiesto que tanto la $\mathrm{CM}(\beta$ no estandarizada $=2.43 \pm .05, r=.801, p<.001)$ como la $\mathrm{CF}$ global $(\beta$ no estandarizada $=.75 \pm .05, r=.373, p<.001)$ se relacionan positivamente con la CVP. Sin embargo, no se encontró una relación estadísticamente significativa entre edad y CVP.

Tabla 1.

Análisis descriptivo de las variables de estudio

\begin{tabular}{|c|c|c|c|c|}
\hline & $\begin{array}{c}\text { Total } \\
(n=1037)\end{array}$ & $\begin{array}{l}\text { Varones } \\
(n=508)\end{array}$ & $\begin{array}{c}\text { Mujeres } \\
(n=529)\end{array}$ & $p^{\mathrm{d}}$ \\
\hline \multicolumn{5}{|l|}{ Calidad de vida percibida (CVP) } \\
\hline Función física (0-100) & $86.2 \pm 15.4$ & $85.8 \pm 16.2$ & $86.5 \pm 14.6$ & .525 \\
\hline Rol físico (0-100) & $90.0 \pm 10.9$ & $89.9 \pm 11.0$ & $90.0 \pm 10.8$ & .860 \\
\hline Dolor corporal $(0-100)$ & $63.7 \pm 13.7$ & $63.8 \pm 13.6$ & $63.5 \pm 13.9$ & .708 \\
\hline Función social $(0-100)$ & $83.6 \pm 12.6$ & $83.4 \pm 11.8$ & $83.8 \pm 13.4$ & .587 \\
\hline Rol emocional (0-100) & $87.6 \pm 10.1$ & $88.4 \pm 10.1$ & $86.8 \pm 10.1$ & $.010 * *$ \\
\hline Salud mental (0-100) & $79.7 \pm 12.5$ & $82.3 \pm 12.4$ & $77.2 \pm 12.1$ & $<.001 * * *$ \\
\hline Vitalidad $(0-100)$ & $78.8 \pm 18.3$ & $78.3 \pm 17.3$ & $79.3 \pm 19.2$ & .377 \\
\hline Salud general (0-100) & $78.6 \pm 18.7$ & $77.1 \pm 17.8$ & $80.0 \pm 19.4$ & $.011 *$ \\
\hline Calidad de vida global $(0-100)^{\mathrm{a}}$ & $80.9 \pm 5.6$ & $80.9 \pm 5.3$ & $80.9 \pm 6.0$ & .898 \\
\hline \multicolumn{5}{|l|}{ Condición física $(\mathrm{CF})$} \\
\hline Fuerza máxima de prensión $(\mathrm{kg})$ & $34.1 \pm 8.6$ & $39.0 \pm 7.9$ & $29.3 \pm 6.2$ & $<.001 * * *$ \\
\hline Abdominales (núm.) & $25.1 \pm 9.4$ & $27.7 \pm 9.1$ & $22.7 \pm 9.0$ & $<.001 * * *$ \\
\hline Ruffier (índice de Ruffier) & $8.91 \pm 3.8$ & $8.1 \pm 3.7$ & $9.7 \pm 3.7$ & $<.001 * * *$ \\
\hline Distancia dedos-plantas $(\mathrm{cm})$ & $-0.4 \pm 6.2$ & $-1.7 \pm 6.4$ & $0.7 \pm 5.7$ & $<.001 * * *$ \\
\hline Condición física global $(0-10)^{\mathrm{b}}$ & $5.3 \pm 1.8$ & $5.5 \pm 1.7$ & $5.1 \pm 1.7$ & $<.001 * * *$ \\
\hline Menor condición física global, $n(\%)$ & $565(54.5)$ & $260(46.0)$ & $305(54.0)$ & .058 \\
\hline Mayor condición física global, $n(\%)$ & $472(45.5)$ & $248(52.5)$ & $224(47.5)$ & .269 \\
\hline \multicolumn{5}{|l|}{ Coordinación motriz (CM) } \\
\hline Carrera de obstáculos (s) & $11.5 \pm 1.6$ & $10.2 \pm .8$ & $12.8 \pm 1.1$ & $<.001 * * *$ \\
\hline Manejo de balón (s) & $12.1 \pm 1.8$ & $11.2 \pm 1.1$ & $13.1 \pm 1.9$ & $<.001 * * *$ \\
\hline Conducción de balón (s) & $17.9 \pm 6.1$ & $14.6 \pm 2.6$ & $21.0 \pm 6.8$ & $<.001 * * *$ \\
\hline Lanzamiento-recepción (núm.) & $35.4 \pm 7.0$ & $39.2 \pm 5.9$ & $31.8 \pm 6.1$ & $<.001 * * *$ \\
\hline Coordinación motriz $(0-10)^{\mathrm{c}}$ & $5.3 \pm 1.7$ & $5.3 \pm 1.7$ & $5.4 \pm 1.9$ & .388 \\
\hline Menor coordinación motriz, $n(\%)^{\mathrm{c}}$ & $528(50.9)$ & $278(52.7)$ & $250(47.3)$ & .223 \\
\hline Mayor coordinación motriz, $n(\%)^{\mathrm{c}}$ & $509(49.1)$ & $230(45.2)$ & $279(54.8)$ & $.030 *$ \\
\hline \multicolumn{5}{|l|}{ Variables antropométricas } \\
\hline Peso $(\mathrm{kg})$ & $64.7 \pm 12.7$ & $67.8 \pm 14.1$ & $61.7 \pm 10.5$ & $<.001 * * *$ \\
\hline Talla (cm) & $167.6 \pm 7.4$ & $171.3 \pm 7.3$ & $164.2 \pm 5.7$ & $<.001 * * *$ \\
\hline Índice de masa corporal $\left(\mathrm{kg} / \mathrm{cm}^{2}\right)$ & $22.9 \pm 3.9$ & $22.9 \pm 4.1$ & $22.8 \pm 3.6$ & 0.734 \\
\hline
\end{tabular}

Nota. ${ }^{\mathrm{a}}$ Media de las variables de la calidad de vida; ${ }^{\mathrm{b}}$ media de los test de CF; ${ }^{\mathrm{c}}$ media de las variables de la CM; ${ }^{\mathrm{d}}$ valores de $p$ para la comparación de sexos (MANOva para variables continuas, test de chi-cuadrado para variables categóricas). ${ }^{*} p<.05$;

$* * p<.01 ; * * * p<.001$. 
Tabla 2.

Asociación entre la calidad de vida percibida, la condición física global y la coordinación motriz

\begin{tabular}{ccccc}
\hline & $\beta$ & EE & $r$ & $p$ \\
\hline Sexo & 0.039 & .19 & .006 & .844 \\
Edad (años) & 0.49 & .08 & .171 & $<.001^{* * *}$ \\
Índice de masa corporal $\left(\mathrm{kg} / \mathrm{m}^{2}\right)$ & 0.04 & .02 & .048 & .121 \\
Condición física global $(0-10)$ & 0.75 & .05 & .373 & $<.001^{* * *}$ \\
Coordinación motriz $(0-10)$ & 2.43 & .05 & .801 & $<.001^{* * *}$ \\
\hline
\end{tabular}

Nota. $\beta=$ beta no estandarizada; $\mathrm{EE}=$ error estándar; $r=$ correlación parcial (Pearson). ${ }^{*} p<.05 ; * * p<.01 ; * * * p<.001$.

Tabla 3.

Análisis de las dimensiones de la calidad de vida percibida según el nivel de condición física global

\begin{tabular}{|c|c|c|c|c|}
\hline \multicolumn{5}{|c|}{ Condición física global ${ }^{\dagger}$} \\
\hline & $\begin{array}{l}\text { Menor } \\
(n=565)\end{array}$ & $\begin{array}{c}\text { Mayor } \\
(n=472)\end{array}$ & $\eta^{2}$ & $F$ \\
\hline $\begin{array}{l}\text { Función física (0-100) } \\
\text { (IC } 95 \%)\end{array}$ & $\begin{array}{c}82.7 \pm 16.7 \\
(81.666-83.981)\end{array}$ & $\begin{array}{c}90.3 \pm 12.5 \\
(88.873-91.406)\end{array}$ & .063 & $69.799 * * *$ \\
\hline $\begin{array}{l}\text { Rol físico }(0-100) \\
\text { (IC } 95 \%)\end{array}$ & $\begin{array}{c}87.0 \pm 11.4 \\
(86.046-87.751)\end{array}$ & $\begin{array}{c}93.6 \pm 9.0 \\
(92.731-94.597)\end{array}$ & .096 & $109.932 * * *$ \\
\hline $\begin{array}{l}\text { Dolor corporal }(0-100) \\
(\text { IC } 95 \%)\end{array}$ & $\begin{array}{c}68.6 \pm 12.3 \\
(67.736-69.774)\end{array}$ & $\begin{array}{c}57.8 \pm 13.1 \\
(56.477-58.707)\end{array}$ & .169 & $209.706^{* * *}$ \\
\hline $\begin{array}{l}\text { Función social }(0-100) \\
\text { (IC } 95 \%)\end{array}$ & $\begin{array}{c}82.1 \pm 13.0 \\
(81.040-83.108)\end{array}$ & $\begin{array}{c}85.3 \pm 12.0 \\
(84.278-86.541)\end{array}$ & .017 & $18.185^{* * *}$ \\
\hline $\begin{array}{l}\text { Rol emocional (0-100) } \\
\text { (IC } 95 \%)\end{array}$ & $\begin{array}{c}86.8 \pm 10.2 \\
(86.024-87.685)\end{array}$ & $\begin{array}{c}88.6 \pm 9.9 \\
(87.594-89.411)\end{array}$ & .007 & $6.887 * *$ \\
\hline $\begin{array}{l}\text { Salud mental (0-100) } \\
\text { (IC } 95 \%)\end{array}$ & $\begin{array}{c}74.4 \pm 10.5 \\
(73.602-75.384)\end{array}$ & $\begin{array}{c}86.0 \pm 11.8 \\
(84.928-86.879)\end{array}$ & .217 & $286.311 * * *$ \\
\hline $\begin{array}{l}\text { Vitalidad (0-100) } \\
\text { (IC 95\%) }\end{array}$ & $\begin{array}{c}77.7 \pm 18.9 \\
(76.100-79.108)\end{array}$ & $\begin{array}{c}80.1 \pm 17.4 \\
(78.523-81.815)\end{array}$ & .005 & $5.078^{*}$ \\
\hline $\begin{array}{l}\text { Salud general (0-100) } \\
\text { (IC } 95 \%)\end{array}$ & $\begin{array}{c}79.5 \pm 19.0 \\
(77.845-80.920)\end{array}$ & $\begin{array}{c}77.6 \pm 18.3 \\
(76.027-79.392)\end{array}$ & .002 & 2.069 \\
\hline $\begin{array}{c}\text { Calidad de vida global (0-100) } \\
\text { (IC } 95 \%)\end{array}$ & $\begin{array}{c}79.7 \pm 5.3 \\
(79.239-80.143)\end{array}$ & $\begin{array}{c}82.4 \pm 5.7 \\
(81.879-82.867)\end{array}$ & .056 & $61.545 * * *$ \\
\hline
\end{tabular}

Nota. Los resultados se presentan como media \pm desviación estándar. IC $=$ intervalo de confianza (límite inferior-límite superior).

${ }^{\dagger}$ Niveles establecidos a partir del percentil $50{ }^{*} p<.05 ; * * p<.01 ; * * p<.001$.

Adicional a esto, como aparece en la Tabla 3, se encuentra que los participantes con mayor $\mathrm{CF}$ global mostraron valores promedio más elevados en función física $(p<.001)$, rol físico $(p<.001)$, función social $(p<.001)$, rol emocional $(p<.01)$, salud mental $(p<.001)$, vitalidad $(p<.05)$ y calidad de vida global $(p<.001)$; mientras que aquellos con menor $\mathrm{CF}$ global obtuvieron puntuaciones superiores en dolor corporal $(p<.001)$.

Y, finalmente, como aparece en la Tabla 4, se encuentra que los participantes con mayor CM mostraron mejores registros en función social, salud general, vitalidad y calidad de vida global $(p<.001)$, mientras que sus semejantes con menor CM presentaron valores más elevados en las variables función física $(p<.01)$, rol físico $(p<.001) \mathrm{y}$ rol emocional $(p<.001)$.

\section{Discusión}

Los resultados de este estudio indican que tanto la $\mathrm{CF}$ como la CM se relacionan de manera positiva con la CVP en adolescentes (véase Tabla 2), lo cual confirma las hipótesis establecidas al momento de plantear el estudio. Estos resultados se mantienen en sintonía con los hallados en la literatura científica, a saber, que el funcionamiento motor interfiere con numerosos aspectos de la vida del adolescente, incluidos los logros académicos y las actividades de ocio (Ortiz \& Ramírez, 2020), y que, por tanto, este afecta el cvP de los adolescentes por el costo físico y emocional de sus esfuerzos por participar con éxito en las actividades físicas diarias (Baceviciene et al., 2019; Becerra et al., 2013; Zwicker et al., 2018). En este sentido, estos hallazgos adquieren 
Tabla 4.

Análisis de las dimensiones de la calidad de vida percibida según el nivel de coordinación motriz

\begin{tabular}{|c|c|c|c|c|}
\hline & \multicolumn{2}{|c|}{ Coordinación motriz $^{\dagger}$} & \multirow[b]{2}{*}{$\eta^{2}$} & \multirow[b]{2}{*}{$F$} \\
\hline & $\begin{array}{l}\text { Menor } \\
(n=528)\end{array}$ & $\begin{array}{c}\text { Mayor } \\
(n=509)\end{array}$ & & \\
\hline $\begin{array}{l}\text { Función física }(0-100) \\
\text { (IC } 95 \%)\end{array}$ & $\begin{array}{c}88.4 \pm 13.7 \\
(86.286-88.767)\end{array}$ & $\begin{array}{c}83.8 \pm 16.7 \\
(83.465-85.993)\end{array}$ & .009 & $9.431 * *$ \\
\hline $\begin{array}{c}\text { Rol físico (0-100) } \\
\text { (IC } 95 \%)\end{array}$ & $\begin{array}{c}91.7 \pm 9.6 \\
(91.017-92.856)\end{array}$ & $\begin{array}{c}88.2 \pm 11.9 \\
(87.009-88.883)\end{array}$ & .033 & $34.928 * * *$ \\
\hline $\begin{array}{l}\text { Dolor corporal }(0-100) \\
\text { (IC } 95 \%)\end{array}$ & $\begin{array}{c}64.3 \pm 13.4 \\
(62.707-65.037)\end{array}$ & $\begin{array}{c}63.0 \pm 14.1 \\
(62.282-64.655)\end{array}$ & $<.001$ & 0.223 \\
\hline $\begin{array}{l}\text { Función social }(0-100) \\
\text { (IC } 95 \%)\end{array}$ & $\begin{array}{c}74.5 \pm 9.5 \\
(73.616-75.094)\end{array}$ & $\begin{array}{c}93.0 \pm 7.6 \\
(92.422-93.927)\end{array}$ & .538 & $1203.951 * * *$ \\
\hline $\begin{array}{l}\text { Rol emocional (0-100) } \\
\text { (IC } 95 \%)\end{array}$ & $\begin{array}{c}90.2 \pm 9.3 \\
(89.312-90.992)\end{array}$ & $\begin{array}{c}84.9 \pm 10.2 \\
(84.107-85.818)\end{array}$ & .064 & $70.809 * * *$ \\
\hline $\begin{array}{l}\text { Salud mental (0-100) } \\
\text { (IC } 95 \%)\end{array}$ & $\begin{array}{c}79.5 \pm 12.5 \\
(78.457-80.557)\end{array}$ & $\begin{array}{c}79.9 \pm 12.4 \\
(78.803-80.942)\end{array}$ & $<.001$ & 0.225 \\
\hline $\begin{array}{c}\text { Vitalidad (0-100) } \\
\text { (IC 95\%) }\end{array}$ & $\begin{array}{c}65.5 \pm 15.3 \\
(64.276-66.397)\end{array}$ & $\begin{array}{c}92.5 \pm 8.2 \\
(91.627-93.788)\end{array}$ & .545 & $1236.095 * * *$ \\
\hline $\begin{array}{l}\text { Salud general }(0-100) \\
\text { (IC } 95 \%)\end{array}$ & $\begin{array}{c}65.8 \pm 16.4 \\
(64.546-66.851)\end{array}$ & $\begin{array}{c}91.9 \pm 9.4 \\
(90.852-93.200)\end{array}$ & .584 & $968.469 * * *$ \\
\hline $\begin{array}{l}\text { Calidad de vida global (0-100) } \\
\text { (IC 95\%) }\end{array}$ & $\begin{array}{c}77.4 \pm 4.5 \\
(76.819-77.544)\end{array}$ & $\begin{array}{c}84.6 \pm 4.1 \\
(84.413-85.151)\end{array}$ & .541 & $816.585^{* * *}$ \\
\hline
\end{tabular}

Nota. Los resultados se presentan como media \pm desviación estándar. IC = intervalo de confianza (límite inferior-límite superior). ${ }^{\dagger}$ Niveles establecidos a partir del percentil $50 .{ }^{*} p<.05 ; * * p<.01 ; * * *<.001$.

gran importancia, debido a que evidencian que, además de fomentar el bienestar psicosocial, la intervención educativa debe enfocarse también en fomentar tratamientos dirigidos a la mejora de las habilidades y capacidades motoras de los estudiantes (Karras et al., 2019; Moral-García et al., 2019).

Ahora bien, al categorizar la muestra según su nivel de CF O CM, los resultados mostraron diferencias entre grupos en las dimensiones de la CVP, así como en su valor global. Por una parte, indican que los adolescentes con mayor CF global presentaron una mejor percepción de sí mismos en las dimensiones de función física, rol físico, vitalidad, función social, rol emocional, salud mental y calidad de vida global (véase Tabla 3); resultados que coinciden con estudios previos con preadolescentes y adolescentes (Gálvez et al., 2015c; García-Rubio et al., 2015; Mota et al., 2012; Rosa-Guillamón et al., 2016; Rosa-Guillamón et al., 2018), por lo cual es posible concluir que el nivel de CF se asocia con la CVP, independientemente del sexo, edad y nivel de actividad física tanto objetivo como percibido (García-Rubio et al., 2015). Y, por otra parte, se encontró que la modificación en el nivel de CF influye en la autopercepción — positiva o negativa- de los jóvenes en algunos indicadores emocionales de la CVP y, consecuentemente, en sus conductas; razón por la cual la CF resalta como un elemento diferenciador del estado de salud tanto objetivo como percibido de la muestra evaluada (Rosa-Guillamón et al., 2018; Rosa-Guillamón et al., 2020).

De hecho, algunos estudios han descrito que un menor nivel de $\mathrm{CF}$ - consecuente de un exceso de peso corporalcondiciona la CVP, debido al patrón cultural de la imagen corporal, ya que para las mujeres el tejido adiposo genera una menor atracción, y para los hombres esta misma distribución constituye una fuente de burla entre sus compañeros (Gálvez et al., 2015b; Rodríguez-Barrera et al., 2016). En consecuencia, se puede afirmar que los estudiantes con sobrepeso $u$ obesidad pueden presentar mayores limitaciones para desarrollar la actividad física, más problemas de salud somáticos, autoevaluación negativa, bullying, estigma social, peor estado de ánimo, peor vida familiar, y síntomas de depresión y ansiedad (Hoedjes et al., 2018).

Asimismo, distintos estudios señalan asociaciones consistentes entre resultados más elevados de $\mathrm{CF}$ a causa de un mayor $\mathrm{VO}_{2}$ máx. e indicadores favorables de $\mathrm{CVP}$, lo cual sugiere que la aptitud cardiorrespiratoria puede ser empleada no solo para alcanzar metas fisiológicas de salud de los adolescentes, sino también para lograr objetivos de bienestar psicológico, emocional y social (Guedes et al., 2017), ya que estudiantes con menor aptitud cardiorrespiratoria experimentan un menor bienestar psicológico cuando son expuestos a eventos críticos de la vida (Marques et al., 2017). 
A su vez, algunos estudios sugieren que la aptitud cardiorrespiratoria —en comparación con la fuerza muscular o el perímetro de cintura- es el indicador de la CF que muestra relaciones más consistentes con todos los dominios de la CVP, de modo que la fuerza explosiva del cuerpo se relacionaría solo con una mayor autonomía y no necesariamente con el bienestar psicológico, el apoyo social o el entorno académico (Andersen et al., 2017; Evaristo et al., 2019; Gálvez et al., 2015b). Por ello, desde el ámbito clínico y educativo, mejorar la aptitud cardiorrespiratoria y el estado de peso corporal podrían ser de especial interés para mejorar el estado de salud —objetivo y percibidode los adolescentes (Nieto et al., 2020; Mota et al., 2012).

Ahora bien, en la Tabla 4 quedó reflejada la relación entre la CM y la CVP, donde se reporta que los adolescentes con mayor CM tienen mejores registros en las dimensiones de función social, vitalidad, salud general y calidad de vida global que sus pares homólogos con menor CM; resultados que se muestran coherentes con los hallados en otros estudios (Caçola \& Killian, 2018; Rigoli \& Piek, 2016), y que reflejan que los adolescentes con menor $\mathrm{CM}$ tienen un mayor riesgo de experimentar autoestima más baja, por lo que pueden desarrollar dificultades secundarias debido a sus limitaciones motoras en la vida diaria (Zwicker et al., 2018). Además, como se ha encontrado en otros estudios, estos estudiantes presentan mayores probabilidades de ser excluidos de las actividades de juego grupal, bajos resultados de autoeficacia percibida, una menor percepción de sí mismos sobre su salud, menor habilidad física, menores relaciones entre pares y una menor confianza en sí mismos, todo lo cual repercute en un menor funcionamiento físico, emocional y social, que, por tanto, se convierte en un factor determinante para su CVP (Karras et al., 2019).

Una variable que puede explicar esta posible asociación es la motivación hacia el ejercicio físico, ya que, según la teoría de la autodeterminación, las personas que están intrínseca y extrínsecamente motivadas en sus regulaciones hacia el ejercicio físico muestran una mayor CVP (García-Cantó et al., 2015; Riiser et al., 2014). De hecho, en una revisión sistemática se encontró que el desarrollo de indicadores de un estilo de vida poco saludable - tales como un bajo nivel de actividad física y CF percibida, no participar en deportes, fumar, consumir alcohol, una dieta desequilibrada, uso de internet durante abundantes horas, así como una mala percepción de la aptitud personal dentro de un ambiente obesogénico- se han asociado con mayores quejas somáticas y psicológicas, lo cual repercute directamente en una menor CVP (Wu et al., 2017).

Finalmente, cabe mencionar que este estudio no se encuentra exento de limitaciones, como lo es la falta de un análisis de las diferencias según el género, que fue un estudio descriptivo y no experimental, y que no se contó con un diseño longitudinal donde se pudiera verificar el efecto de la mejora de la CF y la CM sobre la CVP en los adolescentes. No obstante, los resultados de este trabajo aportan datos novedosos y contribuyen a reforzar las conclusiones de investigaciones previas, donde se sugiere la necesidad de redoblar esfuerzos para promocionar la actividad física con el objetivo de mejorar la CF, la CM y la CVP.

Por otra parte, las fortalezas de este estudio derivan del análisis de distintas variables implicadas en la salud relacionada con el estado de CF medido de manera objetiva, y distintas dimensiones de la calidad de vida, lo que ofrece una visión completa de la salud y la CVP de sujetos en una etapa evolutiva vital en el desarrollo de la persona y, en particular, de la formación de su personalidad.

En conclusión, los resultados de este estudio sugieren que los adolescentes con mayor CF tienen una mejor CVP; que los adolescentes con mayor CM muestran una mejor CVP; y que tener una mayor CF, así como una mejor CM —especialmente esta última-, pueden ser variables predictoras de la CVP en adolescentes españoles de 14 a 17 años. Futuros estudios de intervención podrían corroborar si programas orientados al desarrollo de la CF y la CM pueden inducir mejoras en la CVP en adolescentes a lo largo de su escolarización.

\section{Referencias}

Andersen, J. R., Natvig, G. K., Aadland, E., Moe, V. F., Kolotkin, R. L., Anderssen, S. A., \& Resaland, G. K. (2017). Associations between health-related quality of life, cardiorespiratory fitness, muscle strength, physical activity and waist circumference in 10-year-old children: the ASK study. Quality of life research, 26(12), 3421-3428. https:// doi.org/10.1007/s11136-017-1634-1

Baceviciene, M., Jankauskiene, R., \& Emeljanovas, A. (2019). Self-perception of physical activity and fitness is related to lower psychosomatic health symptoms in adolescents with unhealthy lifestyles. BMC public health, 19(1), 980. https:// doi.org/10.1186/s12889-019-7311-2

Becerra, C. A., Reigal, R. E., Hernández-Mendo, A., \& MartínTamayo, I. (2013). Relaciones de la condición física y la composición corporal con la autopercepción de salud. RICYDE. Revista Internacional de Ciencias del Deporte, 9(34), 305318. http://dx.doi.org/10.5232/ricyde2013.03401

Caçola, P., \& Killian, M. (2018). Health-related quality of life in children with Developmental Coordination Disorder: Association between the PedsQL and KIDSCREEN instruments and comparison with their normative samples. Research in developmental disabilities, 75, 32-39. https:// doi.org/10.1016/j.ridd.2018.02.009 
España-Romero, V., Ortega, F. B., Vicente-Rodríguez, G., Artero, E. G., Rey, J. P., \& Ruiz, J. R. (2010). Elbow Position Affects Handgrip Strength in Adolescents: Validity and Reliability of Jamar, Dynex, and Tkk Dynamometers. Journal of Strength and Conditioning Research, 24(1), 272277. https://doi.org/10.1519/JSC.0b013e3181b296a5

Evaristo, S., Moreira, C., Lopes, L., Oliveira, A., Abreu, S., Agostinis-Sobrinho, C., \& Mota, J. (2019). Muscular fitness and cardiorespiratory fitness are associated with healthrelated quality of life: Results from labmed physical activity study. Journal of Exercise Science \& Fitness, 17(2), 55-61. https://doi.org/10.1016/j.jesf.2019.01.002

Fort-Vanmeerhaeghe, A., Román-Viñas, B., \& Font-Lladó, R. (2017). ¿Por qué es importante desarrollar la competencia motriz en la infancia y la adolescencia? Base para un estilo de vida saludable. Apunts. Medicina de l'Esport, 52(195), 103-112. https://doi.org/10.1016/j.apunts.2016.11.001

Gálvez, A., Rodríguez-García, P. L., Rosa, A., García-Canto, E., Pérez Soto, J. J., Tárraga, M. L., \& Tárraga, P. J. (2015a). Nivel de condición física y su relación con el estatus de peso corporal en escolares. Nutrición Hospitalaria, 31(1), 393-400. https://doi.org/10.3305/nh.2015.31.1.8074

Gálvez, A., Rodríguez-García, P. L., García-Cantó, E., Rosa, A., Pérez-Soto, J. J., Tárraga, P. J., \& Tárraga, L. (2015b). Capacidad aeróbica y calidad de vida en escolares de 8 a 12 años. Clínica e Investigación en Arteriosclerosis, 27(5), 239-245. https://doi.org/10.1016/j.arteri.2015.01.001

Gálvez, A., Rosa, A., García-Cantó, E., Rodríguez-García, P. L., Pérez-Soto, J. J., Tárraga, L., \& Tárraga, P. J. (2015c). Estado nutricional y calidad de vida relacionada con la salud en escolares del sureste español. Nutrición Hospitalaria, 31(2), 737-743. https://doi.org/10.3305/nh.2015.31.2.8468

García-Cantó, E., Rodríguez-García, P. L., Pérez-Soto, J. J., Rosa-Guillamón, A., \& Villalba, F. (2015). Autopercepción de competencia motriz y su relación con la práctica físicodeportiva en escolares de la Región de Murcia (España). REXE. Revista de Estudios y Experiencias en Educación, 14(27), 49-61. https://dialnet.unirioja.es/descarga/articu lo/5287424.pdf

García-Rubio, J., Olivares, P. R., Lopez-Legarrea, P., GomezCampos, R., Cossio-Bolaños, M. A., \& Merellano-Navarro, E. (2015). Asociación entre la calidad de vida relacionada con la salud, el estado nutricional (IMC) y los niveles de actividad física y condición física en adolescentes chilenos. Nutrición Hospitalaria, 32(4), 1695-1702. http://dx.doi. org/10.3305/nh.2015.32.4.9182

Gil, P., Carrillo-López, P. J., García, E., \& Rosa, A. (2020). Incidencia del sexo, el índice de masa corporal y el número de hermanos en la competencia motriz autopercibida. Revista Cubana de Investigaciones Biomédicas, 39(1), 1-14. http://www.revibiomedica.sld.cu/index.php/ibi/ article/view/370/671
Guedes, D. P., Astudillo, H. A., Morales, J. M., Vecino, J. C., Araujo, C. E., \& Pires-Júnior, R. (2017). Aptitud cardiorrespiratoria y calidad de vida relacionada con la salud de adolescentes latinoamericanos. Revista Andaluza de Medicina del Deporte, 10(2), 47-53. https://doi.org/10.1016/j. ramd.2016.02.001

Guillamón, A. R., Cantó, E. G., \& García, H. M. (2020). Motor coordination and academic performance in primary school students. Journal of Human Sport and Exercise, 16(2), 1-14. https://doi.org/10.14198/jhse.2021.162.02

Herranz, A., López de Mesa, R., \& Azcona, C. (2013). Percepción de padres e hijos de la calidad de vida relacionada con la salud de niños según el estado ponderal en Navarra, España. Archivos Argentinos de Pediatría, 111(6), 508-15. https://doi.org/10.5546/aap.2013.508

Hoedjes, M., Makkes, S., Halberstadt, J., Noordam, H., Renders, C. M., Bosmans, J. E., \& Seidell, J. C. (2018). Health-related quality of life in children and adolescents with severe obesity after intensive lifestyle treatment and at 1-year follow-up. Obesity facts, 11(2), 116-128. https://doi. org $/ 10.1159 / 000487328$

Karras, H. C., Morin, D. N., Gill, K., Izadi-Najafabadi, S., \& Zwicker, J. G. (2019). Health-related quality of life of children with Developmental Coordination Disorder. Research in developmental disabilities, 84, 85-95. https:// doi.org/10.1016/j.ridd.2018.05.012

Lázaro-Martínez, C. J. (2011). Hábito de consumo de alcohol y su relación con la condición fisica saludable en adolescentes de la Región de Murcia (Tesis doctoral). Universidad de Murcia, Murcia, España.

Marques, A., Mota, J., Gaspar, T., \& de Matos, M. G. (2017). Associations between self-reported fitness and self-rated health, life-satisfaction and health-related quality of life among adolescents. Journal of Exercise Science \& Fitness, 15(1), 8-11. https://doi.org/10.1016/j.jesf.2017.03.001

Mastrantonio, M. P., \& Coduras, O. (2020). Actividad Física y Calidad de Vida Percibida en usuarios de Centros Deportivos Públicos de Terrassa. Retos, 37, 427-433. https://doi.org/10.47197/retos.v37i37.74166

Montero, I., \& León, O. G. (2002). Clasificación y descripción de las metodologías de investigación en Psicología. Revista Internacional de Psicología Clínica y de la Salud, 2(3), 503-508. https://www.redalyc.org/pdf/337/33720308.pdf

Moral-García, J. E., Agraso, A. D., Pérez, J. J., Rosa, A., Tárraga, M. L., García, E., \& Tárraga, P. J. (2019). Práctica de actividad física según adherencia a la dieta mediterránea, consumo de alcohol y motivación en adolescentes. Nutrición Hospitalaria, 36(2), 420-427. http://dx.doi. org/10.20960/nh.2181

Mota, J., Santos, R. M., Silva, P., Aires, L., Martins, C., \& Vale. S. (2012). Associations Between self-rated health with cardiorespiratory fitness and obesity status among adolescent 
girls. Journal of Physical Activity and Health, 9(3), 378-81. https://doi.org/10.1123/jpah.9.3.378

Nieto, L., García, E., \& Rosa, A. (2020). Valores de Condición Física relacionada con la Salud en adolescentes de 14 a 17 años; relación con el estado de peso. Retos, 37(37), 215221. https://doi.org/10.47197/retos.v37i37.69532

Ortiz, R., \& Ramírez, M. L. (2020). Actividad física, cognición y rendimiento escolar: una breve revisión desde las neurociencias. Retos, 38, 868-878. https://doi.org/10.47197/retos. v38i38.72378

Ramón, I., \& Ruiz-Pérez, L. M. (2015). Adolescence, motor coordination problems and competence. Educacion $X X 1$, 18(2), 189-213. https://doi.org/10.5944/educxx1.14601

Rigoli, D., \& Piek, J. P. (2016). Motor Problems as a Risk Factor for Poorer Mental Health in Children and Adolescents: What Do We Know and Should We Be Screening for Psychological Difficulties in Those with Poor Motor Skills? Current Developmental Disorders Reports, 3(3), 190-194. https://doi.org/10.1007/s40474-016-0091-9

Rigoli, D., Piek, J. P., \& Kane, R. (2012). Motor skills and psychosocial correlates in a normal adolescent sample. Pediatrics, 129(4), 892-900. https://doi.org/10.1542/ peds.2011-1237

Riiser, K., Ommundsen, Y., Småstuen, M. C., Løndal, K., Misvær, N., \& Helseth, S. (2014). The relationship between fitness and health-related quality of life and the mediating role of self-determined motivation in overweight adolescents. Scandinavian Journal of Public Health, 42(8), 766772. https://doi.org/10.1177/1403494814550517

Robinson, L. E., Stodden, D. F. Barnett, L. M., Lopes, V. P., Logan, S. W., Rodrigues, L. P., \& D’Hondt, E. (2015). Motor Competence and its Effect on Positive Developmental Trajectories of Health. Sports Medicine, 45(9), 1273-1284. https://oi.org/10.1007/s40279-015-0351-6

Rodríguez-Barrera, J. C., Bastidas, M., Giuseppe, G., \& OlayaContreras, P. (2016). Calidad de vida relacionada con la salud en escolares de 10 a 14 años con sobrepeso y obesidad en la ciudad de Medellín, Colombia. Universitas Psychologica, 15(2), 301-314. https://doi.org/10.11144/ Javeriana.upsy15-2.cves

Rosa, A., García-Cantó, E., \& Carrillo, P. J. (2018). Percepción de salud, actividad física y condición física en escolares. Cuadernos de Psicología del Deporte, 18(3), 179-189. https://revistas.um.es/cpd/article/view/330901

Rosa, A., García, E., \& Martínez, H. (2020). Análisis de la coordinación motriz global en escolares según género, edad y nivel de actividad física. Retos, 38(38), 95-101. https://doi. org/10.47197/retos.v38i38.73938

Rosa-Guillamón, A. (2017). Análisis bibliográfico de las baterías de evaluación de la condición física. Revista Peruana de Ciencias de la Actividad Física y del Deporte, 4(4), 533-543. https://www.rpcafd.com/index.php/rpcafd/issue/ download/16/13
Rosa-Guillamón, A., García-Cantó, E., \& Pérez-Soto, J. J. (2018). Condición física y bienestar emocional en escolares de 7 a 12 años. Acta Colombiana de Psicología, 21(2), 282291. https://www.dx.doi.org/10.14718/ACP.2018.21.2.13

Rosa-Guillamón, A., García-Cantó, E., Rodríguez-García, P. L., \& Pérez-Soto, J. J. (2016). Condición física y calidad de vida en escolares de 8 a 12 años. Revista de la Facultad de Medicina, 65(1), 37-42. https://doi.org/10.15446/revfac med.v65n1.59634

Rosa-Guillamón, A., Carrillo-López, P. J., \& García-Cantó, E. (2020). Analysis of physical fitness according to sex, age, body mass index and level of physical activity in Spanish elementary school students. Revista de la Facultad de Medicina, 68(1), 92-99. http://dx.doi.org/10.15446/revfacmed.v68n1.69977

Ruiz, J. R., Castro-Pinero, J., Artero, E. G., Ortega, F. B., Sjostrom, M., Suni, J., \& Castillo, M. J. (2009). Predictive validity of health-related fitness in youth: a systematic review. British Journal of Sports Medicine, 43(12), 909-923. https://doi.org/10.1136/bjsm.2008.056499

Ruiz-Pérez, L. M., Navia, J. A., Ruiz, A., Ramón, I., \& Palomo, M. (2016). Motor coordination and academic performance in adolescents. Retos, 29, 86-89. https://doi.org/10.47197/ retos.v0i29.38769

Salinas-Miranda, A. A., Salemi, J. L., King, L. M., Baldwin, J. A., Berry, E. L., Austin D. A., Scarborough, K., Spooner, K. K., Zoorob, R. J., \& Salihu, H. M. (2015). Adverse childhood experiences and health-related quality of life in adulthood: revelations from a community needs assessment. Health and Quality of Life Outcomes, 13(123), 1-12. https:// doi.org/10.1186/s12955-015-0323-4

Salum, G. A., DeSousa, D. A., Bosa, V. L., Schuch, I., Goldani, M., Isolan, L. R., Teche, S. P., Fleck, M. P., Rohde, L. A., \& Manfro, G. G. (2014). Internalizing disorders and quality of life in adolescence: evidence for independent associations. Revista Brasileña de Psiquiatría, 36(4), 305-12. https://doi. org/10.1590/1516-4446-2014-1362

Urzúa, M.A., \& Caqueo-Urizar, A. (2012). Calidad de vida: Una revisión teórica del concepto. Terapia Psicológica, 30(1), 6171. http://dx.doi.org/10.4067/S0718-48082012000100006

Vilagut, G., Ferrer, M., Rajmil, L., Rebollo, P., PermanyerMiralda, G., Quintana, J. M., Santed, R., Valderas, J., Ribera, A., Domingo-Salvany, A., \& Alonso, J. (2005). El cuestionario de salud SF-36 español: una década de experiencia y nuevos desarrollos. Gaceta sanitaria, 19(2), 135150. https://doi.org/10.1157/13074369

Ware, J. E., \& Sherbourne, C. D. (1992). The MOS 36-item short-form health survey (SF-36) (I). Conceptual framework and item selection. Medical Care, 30, 473-83. https://doi. org/10.1097/00005650-199206000-00002

Wu, X. Y., Han, L. H., Zhang, J. H., Luo, S., Hu, J. W., \& Sun, K. (2017). The influence of physical activity, sedentary behavior on health-related quality of life among the general 
106

population of children and adolescents: A systematic review. PloS ONE, 12(11), e0187668. https://doi.org/10.1371/ journal.pone. 0187668

Zwicker, J. G., Suto, M., Harris, S. R., Vlasakova, N., \& Missiuna, C. (2018). Developmental coordination disorder is more than a motor problem: children describe the impact of daily struggles on their quality of life. British Journal of Occupational Therapy, 81(2), 65-73. https://doi. org/10.1177/0308022617735046 